\title{
Which Approach Is Most Optimal for Needle Electromyographic Examination of the Biceps Femoris Short Head: Medial or Lateral?
}

\author{
Jong Heon Park, MD ${ }^{1}$, Im Joo Rhyu, MD, PhD ${ }^{2,3}$, Ha Kyoung Lim, MD ${ }^{4}$, Jae Hyun Cha, MD ${ }^{5}$, \\ Gi Jun Shin, $\mathrm{MD}^{6}$, Hye Chang Rhim, $\mathrm{MD}^{7}$, Dong Hwee Kim, $\mathrm{MD}, \mathrm{PhD}^{7}$ \\ ${ }^{1}$ Department of Dermatology, Korea University Ansan Hospital, Ansan; \\ ${ }^{2}$ Department of Anatomy, Korea University College of Medicine, Seoul; \\ ${ }^{3}$ Division of Brain Korea 21 Plus Program for Biomedical Science, Korea University College of Medicine, Seoul; \\ ${ }^{4}$ Department of Internal Medicine, Korea University Anam Hospital, Seoul; \\ ${ }^{5}$ Department of Laboratory Medicine, Korea University Anam Hospital, Seoul; \\ ${ }^{6}$ Department of Orthopedic Surgery, Korea University Ansan Hospital, Ansan; \\ ${ }^{7}$ Department of Physical Medicine and Rehabilitation, Korea University College of Medicine, Seoul, Korea
}

\begin{abstract}
Objective To investigate the anatomical characteristics of the biceps femoris short head (BS) and determine the optimal needle placement for BS examination.

Methods Twenty-one lower limbs were dissected. The distances from the medial and lateral margins of the biceps femoris long head (BL) tendon to the common fibular nerve (CFN) (M_CFN_VD and L_CFN_VD, respectively) and the distance from the lateral margin of the BL tendon to the lateral margin of the BS (L_BS_HD) were measured 5 $\mathrm{cm}$ proximal to the tip of the fibular head (P1), four fingerbreadths proximal to the tip of the fibular head (P2), and at the upper apex of the popliteal fossa (P3).

Results The BS was located lateral to the BL tendon. The CFN was located along the medial margin of the $\mathrm{BL}$ tendon. The median values were 2.0 (P1), 3.0 (P2), and 0 mm (P3) for M_CFN_VD; and 17.4 (P1), 20.2 (P2), and $21.8 \mathrm{~mm}$ (P3) for L_CFN_VD; and 8.1 (P1), 8.8 (P2), and 13.0 mm (P3) for L_BS_VD.

Conclusion The lateral approach to the BL tendon was safer than the medial approach for examining the BS. Amore proximal insertion site around the upper apex of the popliteal fossa was more accurate than the distal insertion site. In this study, we propose a safer and more accurate approach for electromyography of the BS.
\end{abstract}

Keywords Hamstring muscles, Hamstring tendons, Electromyography, Fibular nerve, Cadaver

Received May 7, 2020; Revised August 18, 2020; Accepted October 6, 2020; Published online February 9, 2021

Corresponding author: Dong Hwee Kim

Department of Physical Medicine and Rehabilitation, Korea University Ansan Hospital, 123 Jeokgeum-ro, Danwon-gu, Ansan 15355, Korea. Tel: $+82-$ 31-412-5330, Fax: +82-31-412-4215, E-mail: rmkdh@korea.ac.kr

ORCID: Jong Heon Park (https://orcid.org/0000-0002-3162-8509); Im Joo Rhyu (https://orcid.org/0000-0002-5558-6278); Ha Kyoung Lim (https:// orcid.org/0000-0002-9677-2165); Jae Hyun Cha (https://orcid.org/0000-0003-2503-3469); Gi Jun Shin (https://orcid.org/0000-0003-3874-2512); Hye Chang Rhim (https://orcid.org/0000-0002-7986-6493); Dong Hwee Kim (https://orcid.org/0000-0002-8116-0078).

(c) This is an open-access article distributed under the terms of the Creative Commons Attribution Non-Commercial License (http://creativecommons.org/ licenses/by-nc/4.0) which permits unrestricted noncommercial use, distribution, and reproduction in any medium, provided the original work is properly cited. Copyright ( 2021 by Korean Academy of Rehabilitation Medicine 


\section{INTRODUCTION}

The biceps femoris muscle is a strong flexor of the knee joint and an extensor of the hip joint. It comprises a long head and a short head innervated by the tibial division and the common fibular division of the sciatic nerve, respectively. The former originates from the posterior portion of the ischial tuberosity, whereas the latter arises from the lateral lip of the linea aspera and the lateral supracondylar line of the femur. The biceps femoris long head (BL) becomes tendinous distally and is joined by the short head, and the round common tendon is inserted in the fibular head. In the distal thigh, the sciatic nerve is divided into the tibial nerve and common fibular nerve (CFN). While the tibial nerve extends straight into the calf bisecting the popliteal fossa, the CFN closely follows the medial margin of the BL tendon and winds around the fibular neck [1-3].

The biceps femoris short head (BS) is clinically relevant as it is the only muscle innervated by the common fibular portion of the sciatic nerve. Several techniques have been proposed for examining the BS using needle electromyography (EMG). Chu-Andrews and Johnson [4] recommended the insertion of a needle $5 \mathrm{~cm}$ above the knee while flexed, just medial or lateral to the BL tendon. Perotto et al. [5] suggested needle insertion at four fingerbreadths proximal to the fibular head, just medial to the BL tendon. Lee and DeLisa [6] proposed inserting the needle immediately lateral or medial to the BL tendon at the level of the popliteal fossa. However, considering the location of the CFN around the distal thigh, the medial insertion approach to the BL tendon for examining the BS could be dangerous during needle EMG. Furthermore, most reports, including those mentioned earlier, do not describe the potential risks of nerve damage during needle insertion. Previous studies that assessed the accuracy of needle placement in the lower limb muscles using EMG demonstrated a high risk of nerve damage and low accuracy rate with BS examination [7-9]. The anatomical characteristics of the BS, including its relative location to the BL tendon, have not been described in detail in anatomy studies $[8,10]$. However, considering that the BS originates at the lateral lip of the linea aspera and the lateral supracondylar line of the femur, the BS should be located on the lateral side of the BL tendon. Consequently, we hypothesized that the lateral approach would be a safer and more accurate method than the medial approach for examining the BS when using needle EMG.

The purpose of this study was to investigate the anatomical characteristics of the BS and to examine the relationship between the CFN and BL tendon. In addition, we aimed to propose an optimal technique for needle placement for EMG of the BS muscle using cadaver dissection.

\section{MATERIALS AND METHODS}

A total of 21 lower limbs (11 right limbs, 10 left limbs) from 12 fresh cadavers ( 7 males, 5 females) were dissected. Two lower limbs had been previously dissected for other studies, and one lower limb was distorted. Therefore, these three limbs were excluded from the study. The Institutional Review Board exempted this cadaveric study from review.

Skin and subcutaneous tissue were first dissected from the ischial tuberosity to the knee. As shown in Fig. 1, the fibular head and ischial tuberosity were marked. Further

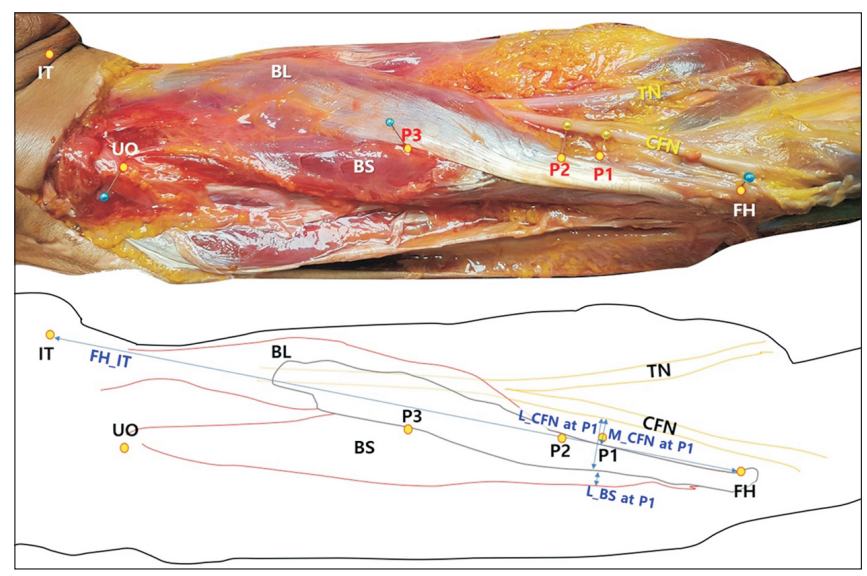

Fig. 1. Dissected cadaver indicating the anatomical marking sites and needle insertion levels according to the three commonly referred methods for electromyography of the biceps femoris short head muscle (BS). The common fibular nerve (CFN) divided from the sciatic nerve follows closely along the medial margin of the biceps femoris long head (BL) tendon. The BS is located laterally along the lateral margin of the $\mathrm{BL}$ tendon. TN, tibial nerve; IT, ischial tuberosity; UO, upper origin of the BS; $\mathrm{FH}$, fibular head; $\mathrm{P} 1,5 \mathrm{~cm}$ proximal to the tip of the $\mathrm{FH}$; P2, four fingerbreadths proximal to the FH (approximately $7 \mathrm{~cm}$ in this study); P3, the upper apex of the popliteal fossa. 
dissections were performed to expose the BL and BS. The upper origin of the BS and the midpoint between its upper origin and insertion were marked.

Needle markings were made at three different sites according to previously reported techniques for needle EMG of the BS [4-6]: $5 \mathrm{~cm}$ proximal to the tip of the fibular head (P1) [4], four fingerbreadths (approximately $7 \mathrm{~cm}$ in this study) proximal to the tip of the fibular head (P2) [5], and at the upper apex of the popliteal fossa where the semimembranosus and biceps femoris muscles meet (P3) [6].

The distances from the fibular head to the ischial tuberosity and to the apex of the popliteal fossa were measured (FH_IT and FH_PF, respectively), and the ratio of FH_PF to FH_IT was calculated. In addition, the distances from the fibular head to the upper origin and to the midpoint of the BS parallel to the axis of the BL tendon (FH_UO

Table 1. Anatomical parameters of the biceps femoris long head and short head

\begin{tabular}{lc}
\hline \multicolumn{1}{c}{ Parameter } & Value \\
\hline FH_IT $(\mathrm{mm})$ & $335.0(279.0-385.0)$ \\
FH_PF $(\mathrm{mm})$ & $150.0(101.0-185.0)$ \\
FH_UO (mm) & $260.0(215.0-290.0)$ \\
FH_MP $(\mathrm{mm})$ & $130.0(107.5-145.0)$ \\
\hline
\end{tabular}

Values are presented as median (min-max).

FH_IT, distance between the fibular head and the ischial tuberosity; FH_PF, distance between the fibular head and the popliteal fossa; FH_UO, distance between the fibular head and the upper origin of the biceps femoris short head muscle; FH_MP, distance between the fibular head and the midpoint of the biceps femoris short head. and FH_MP, respectively) were measured, and the ratios of both parameters to FH_IT were calculated. Finally, the vertical distances from the medial and lateral margins of the $\mathrm{BL}$ tendon to the lateral margin of the CFN (M_CFN VD and L_CFN_VD, respectively) were measured at the P1, P2, and P3 levels. The vertical distance from the lateral margin of the $\mathrm{BL}$ tendon to the lateral margin of the BS (L_BS_VD) was measured at each P level. M_CFN_VD was recorded as a negative value if the $\mathrm{CFN}$ was located lateral to the medial margin of the BL tendon.

The shapes of the BL and BS were also recorded as the unipennate or bipennate type.

All statistical analyses were performed with MedCalc version 19.2.1 (MedCalc Software Ltd, Ostend, Belgium). Nonparametric tests were conducted owing to the small sample size and the non-normal distribution of some parameters; for these, the medians with minimum and maximum values were provided. The Friedman test with further post hoc analysis was used to analyze the differences in M_CFN_VD, L_CFN_VD, and L_BS_VD among the three insertion levels. The distributions of M_CFN VD, L_CFN_VD, and L_BS_VD were analyzed using a box and whisker plot. A p-value of 0.05 was considered statistically significant.

\section{RESULTS}

\section{Morphological data of the biceps femoris short head}

The anatomical characteristics of the BS and BL are shown in Table 1. The median FH_IT value, which was similar to the thigh length, was $335.0 \mathrm{~mm}$. The median FH_UO (the BS length) and FH_MP values were 260.0

Table 2. Horizontal distances from the BS and CFN to the BL tendon using various needle insertion techniques for the examination of the BS muscle

\begin{tabular}{lcccc}
\hline \multicolumn{1}{c}{ Parameter } & P1 level & P2 level & P3 level & p-value \\
\hline L_BS_VD $(\mathrm{mm})$ & $8.1(0-18.0)$ & $8.8(0-20.5)$ & $13.0^{\text {a) }}(5.4-30.0)$ & $<0.00001$ \\
L_CFN_VD $(\mathrm{mm})$ & $17.4^{\text {b) }}(8.4-24.5)$ & $20.2(10.2-31.9)$ & $21.8(10.2-49.5)$ & 0.00003 \\
M_CFN_VD $(\mathrm{mm})$ & $2.0(-5.0-11.2)$ & $3.0(-6.0-13.0)$ & $0^{\text {a) }}(-12.5-42.0)$ & 0.01167
\end{tabular}

Values are presented as median (min-max).

BS, biceps femoris long head; CFN, common fibular nerve; BL, biceps femoris long head; P1 level, $5 \mathrm{~cm}$ proximal to the tip of the fibular head; P2 level, four fingerbreadths proximal to the fibular head (approximately $7 \mathrm{~cm}$ in this study); P3 level, upper apex of the popliteal fossa; L_ BS_VD, vertical distance from the lateral margin of the BL tendon to the lateral border of the BS; L_CFN_VD, vertical distance from the lateral border of the BL tendon to the CFN; $\mathrm{M}_{-}$ CFN_VD, vertical distance from the medial margin of the BL tendon to the CFN.

${ }^{a)} \mathrm{p}<0.05$ on comparison with the P1 and P2 levels.

${ }^{b)} \mathrm{p}<0.05$ on comparison with the P2 and P3 levels. 
and $130.0 \mathrm{~mm}$, respectively. The mean ratio of the BS length to the thigh length was $0.77 \pm 0.04$. In addition, the mean ratio of the $\mathrm{BS}$ midpoint to the thigh length was $0.38 \pm 0.02$. Morphologically, the BL was of the unipennate type and located along the medial side of the BL tendon (Fig. 1). The BS was placed on the lateral side of the BL tendon and had a convergent shape. The medial side of the BS was attached to the $\mathrm{BL}$; thus, distinguishing the two muscles was difficult.
Relationship between the BL tendon and the CFN and BS

The median FH_PF value, designated as P3, was 150.0 $\mathrm{mm}$, and its ratio to the thigh length was 0.42 . The median M_CFN_VD values were 2.0 (P1), 3.0 (P2), and $0 \mathrm{~mm}$ (P3). The median L_CFN_VD values were 17.4 (P1), 20.2 (P2), and $21.8 \mathrm{~mm}$ (P3) (Table 2). Significant differences in M_CFN_HD and L_CFN_HD were found among the 3 levels ( $\mathrm{p}=0.01167$ and $\mathrm{p}=0.00003$, respectively) (Table 2). Post-hoc analyses demonstrated that the M_CFN_VD
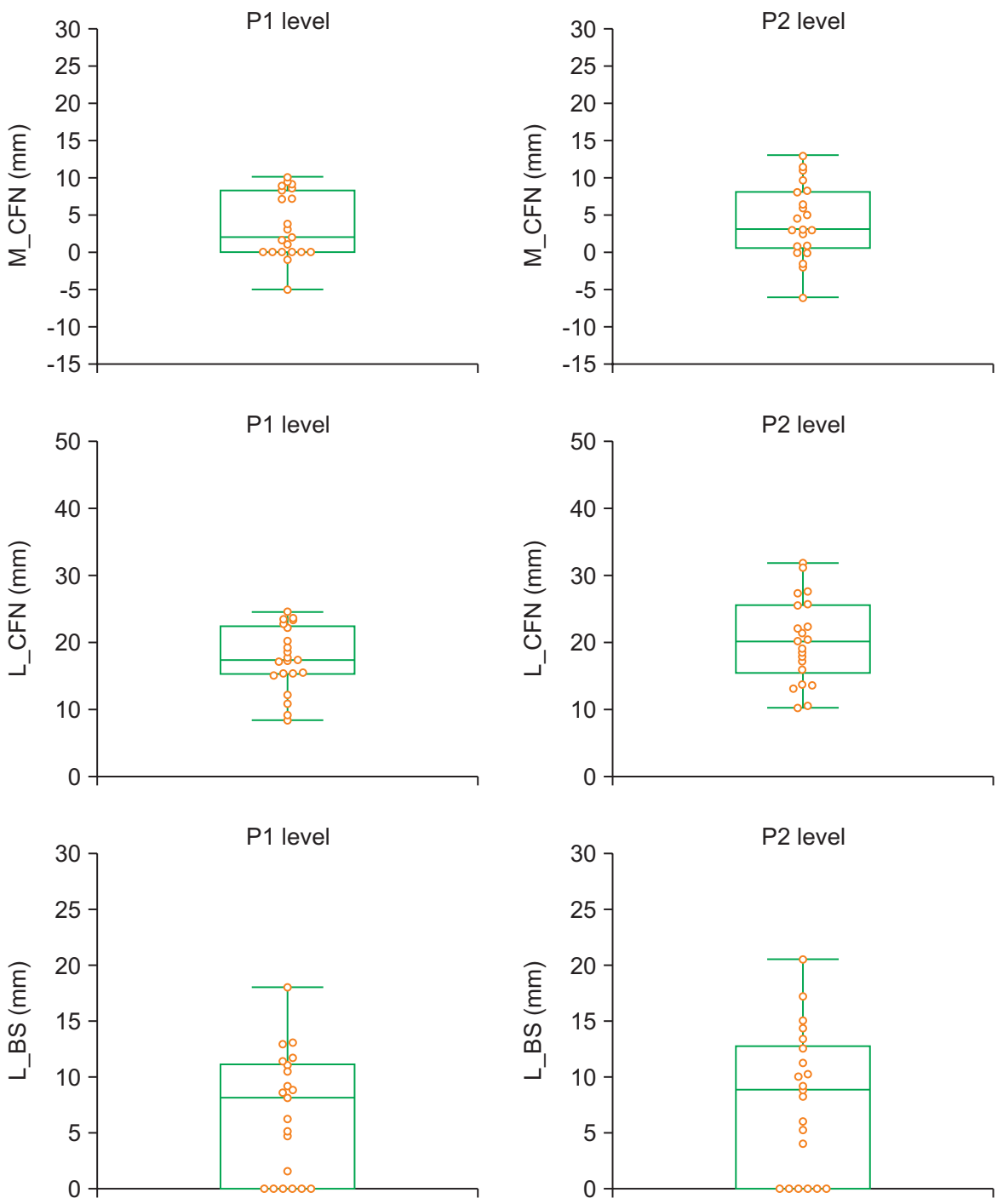
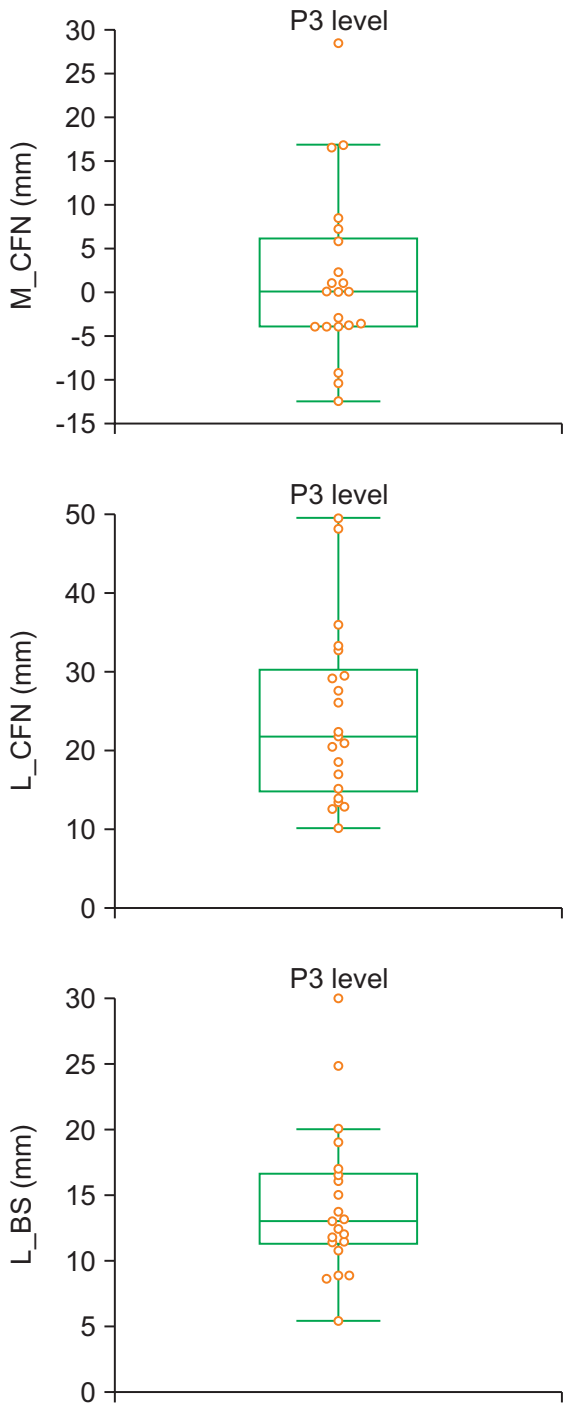

Fig. 2. Box-and-whisker plots of the vertical distance from the lateral margin of the biceps femoris long head (BL) tendon to the lateral margin of the biceps femoris short head (BS) (L_BS_VD), and of the vertical distances from the lateral and medial margins of the BL tendon to the common fibular nerve (CFN) (L_CFN_VD and M_CFN_VD, respectively) at the P1, P2, and P3 levels in all cases (21 limbs). Distances were recorded as a negative value if the CFN was located lateral to the lateral or medial margin of the BL tendon, or if the lateral margin of the BS was located lateral to the lateral margin of the BL tendon. P1 level, $5 \mathrm{~cm}$ proximal to the tip of the fibular head; P2 level, 4 fingerbreadths proximal to the fibular head (approximately $7 \mathrm{~cm}$ in this study); P3 level, upper apex of the popliteal fossa. 
was smaller at the P3 level than at the P1 and P2 levels and that the L_CFN_VD was smaller at the P1 level than at the P2 and P3 levels. Fig. 2 shows that the numbers of M_CFN_VD cases within $10 \mathrm{~mm}$ of the medial margin of the BL tendon were all $21(100 \%)$ at the P1 level and 18 (85.7\%) at the P2 and P3 levels. However, the number of L_CFN_VD cases within $10 \mathrm{~mm}$ of the lateral margin of the BL tendon was $2(9.5 \%)$ at the P1 level and $0(0 \%)$ at the P2 and P3 levels.

The median L_BS_VD values were 8.1, 8.8, and 13.0 $\mathrm{mm}$ at the P1, P2, and P3 levels, respectively. Significant differences were found between each of the 3 levels $(\mathrm{p}<0.00001)$ (Table 2). The post hoc analyses revealed that the L_BS_VHD was larger at the P3 level than at both the P1 and P2 levels. The number of the L_BS_VD cases within $10 \mathrm{~mm}$ of the lateral margin of the BL tendon among all the cases was $14(66.7 \%)$ at the P1 level, $13(61.9 \%)$ at the P2 level, and 4 (19.0\%) at the P3 level. However, the M_BS_VD could not be measured as the medial margin of the BS was overlapped by the muscle and tendon of the BL.

\section{DISCUSSION}

Although needle EMG is useful for identifying nerve injuries, iatrogenic needle injuries still occur. The risk of iatrogenic injuries may be low in routine examinations of commonly selected muscles, but when unfamiliar or deep muscles are evaluated, the risk may increase $[7,8]$. The BS is one of the muscles rarely evaluated via needle EMG. However, the needle EMG of the BS is essential to differentiate common fibular neuropathy around the fibular neck from more proximal lesions.

Several techniques have been used for needle insertion in the BS over several decades [4-6,11]. These studies recommended needle insertion in a location medial or lateral to the BL tendon, three or four fingerbreadths proximal to the lateral knee, $5 \mathrm{~cm}$ above the bend of the knee, or at the level of the superior crease of the popliteal fossa.

Previous studies that used cadavers showed varying degrees of needle placement accuracy according to the characteristics of each muscle including muscle location, size, depth, and local anatomy [7-9]. Haig et al. [7] reported that needle insertion in the BS had a low accuracy, and accurate targeting occurred in approximately
$30 \%$ of the cases; meanwhile, caution-related mistakes such as CFN injury occurred in $20 \%$ of the cases. Another cadaveric study demonstrated similar results (accuracy, $25 \%$; caution-related mistakes, $18.8 \%$ ) [8]. The authors of these studies used the methods developed by Delagi and Perotto [10] and Geiringer [11]. The former recommended the medial approach, and the latter recommended the medial or lateral approach. However, the authors did not report which approach (medial or lateral) was used for the Geiringer's method was used in their studies $[7,8]$. Yun et al. [9] showed that the accuracy of needle insertion in the BS was $68.75 \%$, which was relatively low as compared with those of blind needle insertion in the peroneus longus (75\%) and BL (93.75\%). These low accuracy and high risk rates related to the needle insertion in the BS may be attributed to use of the medial approach or the lack of consistency in needle placement during BS examination. Considering these results, the optimal approach to obtain safe and accurate results must be identified.

The CFN was located along the medial margin of the BL tendon. The median horizontal distance from the medial margin of the BL to the CFN was $\leq 3.0 \mathrm{~mm}$ at all three levels (P1, P2, and P3 levels) (Table 2). As shown in Fig. 2 , in $\geq 85 \%$ of cases, the CFN was located within $10 \mathrm{~mm}$ of the medial margin of the BL tendon. The muscle belly of the BL was located medial to the medial margin of the $\mathrm{BL}$ tendon as the unipennate type. These observations provide evidence of a high risk of CFN injury and the low accuracy of needle placement in the BS during needle EMG using the medial approach. Although none of the studies on needle EMG of the BS have commented on the risk of CFN injury during needle insertion medial to the medial margin of the BL tendon, needle insertion medial to the BL tendon should be avoided as it may increase the risk of CFN injury and decrease the accuracy of needle placement.

On using the lateral approach for examining the $\mathrm{BL}$ tendon, the median horizontal distances from the lateral margin of the BL tendon to the CFN were $\geq 17 \mathrm{~mm}$ at all three levels (P1, P2, and P3 levels) (Table 2). The percentage of cases in which the CFN was located within $10 \mathrm{~mm}$ of the lateral margin of the BL tendon was $9.5 \%$ at the $\mathrm{P} 1$ level and $0 \%$ at the P2 and P3 levels. Morphological examination showed that the BS originated from the lateral lip of the linea aspera, and the lateral supracondylar line 
of the femur was observed to be located lateral to the lateral margin of the BL tendon. The median horizontal distances between the lateral margin of the $\mathrm{BS}$ and $\mathrm{BL}$ tendon were significantly increased at higher levels, and post-hoc analyses revealed a larger value at the P3 level than at the P1 and P2 levels (Table 2). Furthermore, the percentage of cases in which the lateral margin of the BS was located within $10 \mathrm{~mm}$ of the lateral margin of the $\mathrm{BL}$ tendon was $66.7 \%$ at the P1 level, $61.9 \%$ at the P2 level, and $19.0 \%$ at the P3 level (Fig. 2). These findings were compatible with the results of more detailed analyses of "missed" needle placement in BS examinations in previous studies $[7,8]$. In these studies, the needle was often inserted at a location too distal to the muscle belly, and a more proximal needle insertion was recommended. In our study, the P3 level, which is a more proximal site, was thought to be the best location for needle placement in BS examination. The median value of the P3 level (the upper apex of the popliteal fossa) was $150.0 \mathrm{~mm}$ and its ratio to the thigh length (the distance from the fibular head to the ischial tuberosity) was 0.42. At the P3 level, the muscle mass of the BS could be observed in all the cases on the lateral margin of the BL tendon (Fig. 2). Considering that the midpoint of the BS was $130.0 \mathrm{~mm}$ from the fibular head and ratio of the midpoint of the BS to the thigh length was 0.38 , proximal needle placement using the lateral approach would be preferred for accuracy and safety.

The BS muscle is clinically relevant because it is the only muscle innervated by the common fibular portion of the sciatic nerve. High sciatic nerve lesions affecting the common fibular division can cause foot drop in patients, mimicking what is observed in distal peroneal neuropathy at the fibular head [12]. In this case, needle EMG to examine the BS muscle is essential to localize the lesion. Patients with high sciatic nerve lesions and peroneal neuropathy at the fibular head show abnormal findings on fibular nerve conduction studies, whereas those with high sciatic lesions only show an abnormal EMG pattern on evaluation of the BS. Thus, for patients presenting with foot drop or weakness in ankle dorsiflexor muscles, needle EMG of the BS must be performed [13]. Accordingly, a safe and accurate approach for needle placement must be identified to examine the BS. Despite previous studies recommending either the medial or lateral approach, our results suggest that the lateral approach should be preferred to increase the accuracy of measurement and reduce the risk of CFN injury.

Moreover, the site of the EMG needle insertion may serve as a guide for blind injections. In cases of flexed knee spasticity, large muscles of the posterior thigh such as the $\mathrm{BL}$, semimembranosus muscle, or semitendinosus muscle are often targeted for botulinum toxin injection; however, as an alternative, the BS can be injected under ultrasonographic guidance or by using the blind technique $[14,15]$. When using the blind technique for administrating botulinum toxin injections, especially for flexed knee spasticity, our results can be useful in terms of increasing the success rate and lowering the rate of complications associated with misplaced injections.

Our study has several limitations. First, the number of dissected cadavers was small, although fresh cadavers were used. Second, an ultrasonographic technique was not performed for comparative purposes, and the actual relationships of the CFN and BS to the BL tendon could not be analyzed. In addition, because needle placement was not performed according to the techniques or insertion levels in the cadavers, the actual targeting rate and the actual risk of CFN injury could not be measured in this study. As the purpose of this study was to determine the optimal needle insertion site for examining the BS, only the needle placement levels and the directions of the approaches were compared. Further studies using ultrasonography in healthy subjects are needed to evaluate the real relationship of the CFN and BS to the BL tendon.

As the BS shows high clinical relevance in terms of differentiating between the sites of peroneal nerve lesions above or below the knee, it should be examined using accurate and safe techniques. However, previous needle placement techniques for BS examination did not consider the anatomical characteristics of the BS and CFN in relation to the BL tendon. This lack of consideration may account for the low accuracy rate and the high risk of CFN injury associated with needle EMG of the BS. This cadaveric study demonstrated that the lateral approach for examining the $\mathrm{BL}$ tendon was safer than the medial approach. In addition, the more proximal insertion site around the upper apex of the popliteal fossa was more accurate than the distal insertional site. 


\section{CONFLICT OF INTEREST}

No potential conflict of interest relevant to this article was reported.

\section{ACKNOWLEDGMENTS}

This work was supported by a grant from the Korea University (No. K1804221).

\section{AUTHOR CONTRIBUTION}

Conceptualization: Kim DH. Methodology: Park JH, Lim HK, Cha JH, Shin GJ. Formal analysis: Park JH, Lim HK. Funding acquisition: Kim DH. Visualization: Park JH, Kim DH. Writing - original draft: Park JH. Writing review and editing: Rhyu IJ, Rhim HC, Kim DH. Approval of final manuscript: all authors.

\section{REFERENCES}

1. Tubbs RS, Caycedo FJ, Oakes WJ, Salter EG. Descriptive anatomy of the insertion of the biceps femoris muscle. Clin Anat 2006;19:517-21.

2. Standring S. Gray's anatomy: the anatomical basis of clinical practice. 40th ed. Edinburgh, UK: Churchill Livingstone; 2008. p. 1377-8.

3. Moore KL, Dalley AF, Agur AM. Clinically oriented anatomy. 8th ed. Philadelphia, PA: Lippincott Williams \& Wilkins; 2018. p. 731.

4. Chu-Andrews J, Johnson RJ. Electrodiagnosis: an anatomical and clinical approach. Philadelphia, PA: Lippincott; 1986. p. 179.

5. Perotto AO, Delagi EF, Iazzetti J, Morrison D. Anatomical guide for the electromyographer: the limbs and trunk. 4th ed. Springfield, IL: Charles C Thomas;
2005. p. 230-1

6. Lee HJ, DeLisa JA. Manual of nerve conduction study and surface anatomy for needle electromyography. 4th ed. Philadelphia, PA: Lippincott Williams \& Wilkins; 2005. p. 233.

7. Haig AJ, Goodmurphy CW, Harris AR, Ruiz AP, Etemad J. The accuracy of needle placement in lowerlimb muscles: a blinded study. Arch Phys Med Rehabil 2003;84:877-82.

8. Goodmurphy C, Chiodo A, Haig A. The accuracy of needle placement in extremity muscles: a blinded study. J Clin Neurophysiol 2007;24:366-78.

9. Yun JS, Chung MJ, Kim HR, So JI, Park JE, Oh HM, et al. Accuracy of needle placement in cadavers: nonguided versus ultrasound-guided. Ann Rehabil Med 2015;39:163-9.

10. Delagi EF, Perotto A. Anatomic guide for the electromyographer. 2nd ed. Springfield, IL: Charles C Thomas; 1980. p. 183-4.

11. Geiringer SR. Anatomic localization for needle electromyography. Philadelphia, PA: Hanley \& Belfus; 1994. p. 122-3.

12. Katirji B, Wilbourn AJ. High sciatic lesion mimicking peroneal neuropathy at the fibular head. J Neurol Sci 1994;121:172-5.

13. Stewart JD. Focal peripheral neuropathies. West Vancouver, Canada: JBJ Publishing; 2010. p. 472-509.

14. Esquenazi A, Alfaro A, Ayyoub Z, Charles D, Dashtipour K, Graham GD, et al. OnabotulinumtoxinA for lower limb spasticity: guidance from a Delphi panel approach. PM R 2017;9:960-8.

15. Kaymak B, Kara M, Tok F, Ulasli AM, Ozturk GT, Chang KV, et al. Sonographic guide for botulinum toxin injections of the lower limb: EUROMUSCULUS/ USPRM spasticity approach. Eur J Phys Rehabil Med 2018;54:486-98. 